\title{
LA EVALUACIÓN DEL CUELLO EN EL EXAMEN PRECOMPRA DEL CABALLO: REVISIÓN CRÍTICA
}

The evaluation of the neck in the pre-purchase horse exam: A critical review

Morales-Briceño, A.*; Méndez-Sánchez, A.; Pérez-Arévalo, J.

Departamento de Anatomía y Anatomía Patológica Comparadas. Edificio de Sanidad Animal, Campus de Rabanales Ctra. de Madrid km 396, 14071, Córdoba Universidad de Córdoba, España.

* Autor para correspondencia: Morales-Briceño A Telefono: 0034619307223. Email: aamorales13@gmail.com

Historial del artículo:

Recibido: 7 de febrero, 2020

Aceptado: 29 de junio, 2021

\section{RESUMEN}

Se plantea como objetivo describir la evaluación del cuello en el examen precompra del caballo, una revisión crítica. Las 5 etapas recomendadas para el examen precompra son: La primera etapa el examen físico. La segunda etapa consiste en la evaluación al caminar y trotar. La tercera etapa corresponde a la fase de ejercicio vigoroso. La cuarta etapa comprende la fase de reposo. La quinta etapa consiste en una segunda fase de ejercicio. Los exámenes auxiliares complementarios son: la evaluación hematológica, química y serológia. Estudio radiográfico: hallazgos radiológicos de las 14RX de rutina. Endoscopia: se realiza principalmente para estudiar la garganta del caballo. La evaluación del cuello del caballo en el examen precompra se describe a continuación, así como diferentes métodos no invasivos: examen físico de la región del cuello mediante la inspección detallada y la palpación. El examen morfológico se realiza en conjunto con la evaluación del peso corporal mediante la cincha torácica y la condición corporal de acuerdo al protocolo de adiposidad descrito para equinos. La distancia y la circunferencia del cuello se debe medir con una cinta métrica. Entre los métodos invasivos podemos mencionarel Aspirado con Aguja Fina, esta técnica representa una herramienta para la valoración de la región dorsal del cuello. La técnica de biopsia modificada de la región dorsal del cuello en équidos consiste en la toma de muestras mediante un sistema de biopsia ajustable. En conclusión describimos los métodos recomendados y las herramientas disponibles para la evaluación del cuello en el examen precompra de caballos, mediante una revisión crítica.

Palabras claves: caballo, cresta del cuello, cuello, precompra. 


\begin{abstract}
The aim of this study was to describe the assessment of the neck in the pre-purchase examination of the horse a critical review. The 5 steps recommended for the pre-purchase exam are: The first stage the physical examination. The second stage consists of walking and jogging assessment. The third stage corresponds to the vigorous exercise phase. The fourth stage comprises the rest phase. The fifth stage consists of a second phase of exercise. The complementary auxiliary exams are: hematological, chemical and serological evaluation. Radiographic study: radiological findings of 14 routine RX. Endoscopy: is mainly done to study the throat of the horse. The evaluation of the neck of the horse in the pre-buy exam is described below as non-invasive methods: physical examination of the neck region through detailed inspection and palpation. The morphological examination is performed in conjunction with the assessment of body weight using the chest strap and body condition according to the adiposity protocol described for equines. The distance and the circumference of the neck should be measured with a tape measure. Among the invasive methods we can mention Fine Needle Aspiration, this technique represents a tool for the assessment of the dorsal neck region. The modified biopsy technique of the dorsal neck region in equidae consists of sampling using an adjustable biopsy system. In conclusion we describe the recommended methods and tools available for neck evaluation in the pre-purchase horse test, a critical review.
\end{abstract}

Key Words: cresty, horse, neck, pre-purchase.

\section{INTRODUCCIÓN}

En los últimos años ha tenido gran auge en los veterinarios especialistas en equinos el Examen Precompra, dada las implicaciones económicas y legales que representa la compra de un caballo, tanto para el vendedor como para el comprador, en donde el veterinario especialista juega un rol fundamental objetivo, basado en un examen clínico y la ayuda de herramientas diagnosticas. En muchos países en un requisito obligatorio para la compra y venta de un caballo, así como es común en las grandes subastas a nivel mundial la presentación del caballo con su respectivo dossier de la evaluación veterinaria. El verdadero propósito de un examen previo a la compra es "ayudar a proporcionar al comprador suficiente información para tomar una decisión informada sobre si un caballo puede satisfacer sus necesidades", dice Schofield, DVM, Hagyard Equine Medical Institute en Lexington, $\mathrm{Ky}^{1}$. "Estamos allí para evaluar la salud general, la conformación y la solidez para el uso previsto ${ }^{1}$. El examen precompra pretende dar al comprador una opinión veterinaria sobre la aptitud del caballo para el uso previsto, éste puede servir también para obtener las coberturas de un seguro equino ${ }^{2}$
El examen precompra no está destinado a ser un obstáculo de aprobación o no de la compra o venta de un caballo. Realmente es un conjunto de datos objetivos que le ayuda al comprador a tomar la decisión más informada sobre su compra ${ }^{3}$. Recientemente en el congreso de la Asociación de Veterinarios Especialistas en Équidos de España (A.V.E.E), elaboro una guía Ibérica (España-Portugal), de los hallazgos radiológicos de las 14RX que aparecen en el reciente creado modelo de Precompra Estándar de la A.V.E.E. ${ }^{4}$. Durante un examen de pre-compra, un veterinario evalúa a fondo el estado físico del caballo y determina si será útil para el comprador ${ }^{5}$. Al mismo tiempo, el veterinario está buscando problemas futuros que podrían limitar la carrera del caballo como problemas ocultos en una articulación. El papel del veterinario es bastante sencillo: evaluar la utilidad del animal y determinar si el caballo está físicamente sano ${ }^{5}$. En algunos casos, el veterinario que realiza el examen de pre-compra o una compañía de seguros puede recomendar o requerir radiografías adicionales (radiografías), ecografías o un examen endoscópico de los pulmones y la garganta ${ }^{6}$. Estos sólo pueden ser realizados con el consentimiento del propietario actual y se incurrirá en un gasto adicional, así como 
alguna compañía de seguros puede solicitar un conjunto completo de rayos $\mathrm{X}$ si el caballo debe ser asegurado por encima de un cierto valor. En general, un examen precompra incluirá tres fases: Evaluación básica de salud, incluyendo historia de salud, temperatura, pulso, respiración, estado general y conformación. Evaluación de la cojera, incluyendo pruebas de flexión, palpación de los tejidos blandos y evaluación del movimiento. Diagnóstico auxiliar que podría no entrar en juego, incluyendo radiografías (rayos $\mathrm{X})$, ultrasonido, resonancia magnética (MRI) y analítica de sangre ${ }^{1}$. Estudio radiográfico: hallazgos radiológicos de las 14RX de rutina que incluyen: los 4 cascos, articulación metacarpofalangiana (derecha e izquierda), articulación del carpo (derecha e izquierda), articulación metatarso-falangiana (derecha e izquierda), corvejones y la articulación fémur-tibia-rotuliana. Endoscopia: se realiza principalmente para estudiar las vías aéreas superiores del caballo, en algunos casos se puede disponer de la endoscopia de las vías aéreas superiores, durante el ejercicio. Cada fase del proceso puede incluir varios pasos. En algunas circunstancias, un examen en dos etapas puede ser adecuado, por ejemplo para caballos jóvenes que aun no han sido sometido a doma y/o entrenamiento, así como en los casos donde no se disponen de registros clínicos previos, por lo que el veterinario formará una opinión basada en un conjunto restringido de hallazgos objetivos al momento de la revisión, siempre de mutuo acuerdo entre el comprador y el vendedor acepta que confirma que usted entiende y acepta que en un examen limitado, no puede revelar ciertas condiciones que pueden haber sido descubiertas durante el curso de examen completo de dos etapas o más ${ }^{6}$. A continuación se describen las etapas recomendadas para el examen precompra (Figura 1)".

- Etapa 1: Examen físico: El caballo será revisado cuidadosamente con su respectiva reseña y tarjeta de identificación, pasaporte y escaneado de su respectivo microchip en el lado izquierdo del cuello. Los ojos se examinan empleando el oftalmoscopio y se ausculta el corazón a ambos lados del tórax. Se evalúa la dentadura: los dientes delanteros (incisivos) y se calcula la edad, junto con un examen detallado de los dientes de lobo y los bordes afilados de los dientes. El caballo se revisa para detectar aumentos de volumen y cicatrices incluyendo melanomas y sarcoides, y estos serán registrados en su reporte de examen.

- Etapa 2: Evaluación al caminar y trotar: Esta fase incluye una evaluación del caballo al paso y al trote en una línea recta en una superficie dura y nivelada, así como en la estocada en superficies blandas y duras, si las instalaciones son adecuadas y lo permiten. Es una prueba dura para la mayoría de los caballos y se mostrará algún tipo de claudicación o cojera que no puede ser evidente en otros lugares, sin embargo, si la superficie o el temperamento del caballo no es adecuado, esta prueba puede tener que ser omitida, previo acuerdo de las partes interesadas (vendedor/comprador). También se realizan pruebas detalladas de flexión en esta etapa. Una prueba de flexión se considera positiva si el caballo toma más de 3-5 pasos para corregir a una marcha normal. Una prueba de flexión positiva podría indicar problemas con articulaciones o estructuras de tejidos blandos y se considerará junto con el resto del examen.

- Etapa 3: Fase de ejercicio: Se le pide al caballo que camine, trote y galope bajo la silla de montar. El objetivo es que el caballo realice ejercicio vigoroso para aumentar el ritmo cardiaco y respiratorio con el fin de evaluar la forma en que se mueve el caballo, cualquier comportamiento anormal debajo de la silla de montar y cualquier ruido respiratorio que pueda estar asociado con un mal rendimiento.

- Etapa 4: Fase de reposo: El caballo es devuelto al establo y el corazón y los pulmones son evaluados mediante la auscultación y escuchados cuando el caballo se recupera 
de la fase de ejercicio. El comportamiento normal del caballo en el establo también se observa para ver si tiene algún vicio, o para ver si alguna rigidez inducida por el ejercicio se hace evidente.

- Etapa 5: Segunda Fase de Ejercicio: Un segundo examen del caballo al trote se debe realizar. Esto es principalmente para comprobar que el ejercicio extenuante no ha exacerbado un sutil problema subyacente de cojera. Las pruebas de flexión o el trote en un círculo de pequeño diámetro se repiten a veces en este momento. Exámenes auxiliares: Muestra de sangre: la muestra de sangre para la evaluación hematológica general, así como para el descarte de enfermedades de tipo parasitarias y virales como Tripanosoma evansi, Anemia Infecciosa Equina, es común en caballos que partici- pan en competiciones internacionales. Estudio radiográfico: con el objeto de interpretar en detalle los hallazgos radiológicos de las 14RX de rutina en el examen precompra. Endoscopia: se realiza principalmente para estudiar la garganta del caballo.

La región del cuello es una importante área anatómica y fisiológica por sus múltiples connotaciones anatomo-clínicas ${ }^{7}$. En la actualidad la región dorsal del cuello ha sido ampliamente estudiada en los caballos debido a su correlación con el síndrome metabólico equino y obesidad en el caballo ${ }^{7}$. El caballo de deporte presenta un número importante de enfermedades que tienen su origen en la región cervical ${ }^{8}$. En virtud de esta importante área de estudio se plantea como objetivo realizar una revisión crítica de la evaluación del cuello en el examen precompra del caballo.

Figura 1. Algoritmo para la evaluación del cuello del caballo en el examen precompra.

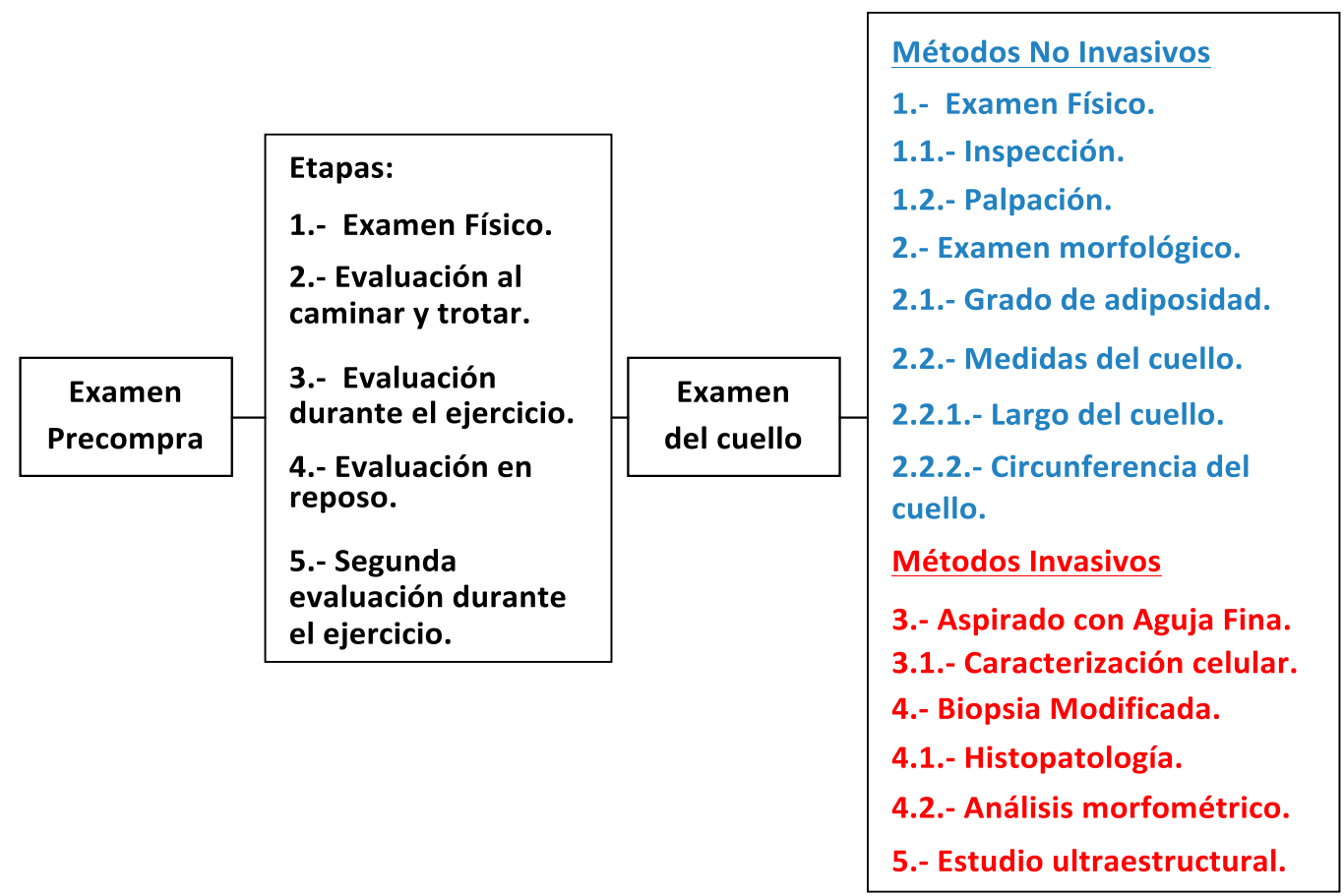




\section{EVALUACIÓN DEL CUELLO}

\section{Métodos no invasivos}

Examen físico: A cada équido se le debe realizar un examen clínico en general con énfasis en la región del cuello mediante la inspección detallada y la palpación siguiendo el protocolo descrito para equinos ${ }^{9}$. También debe realizar el peso corporal y la edad. Considerar aumentos de volumen en la tabla del cuello, cicatrices o alguna anormalidad. Se recomienda la evaluación en estación y en movimiento, durante el movimiento se puede evidenciar el movimiento de la cresta del cuello.

Examen Morfológico: el examen morfológico se realiza en conjunto con la evaluación del peso corporal mediante la cincha torácica, o de ser posible a través de una balanza digital, y la condición corporal de acuerdo al protocolo de adiposidad descrito para equinos ${ }^{9,10}$.

- Grado 0: No hay apariencia visual de cresta (Tejido sobre el ligamento de la nuca). No hay cresta palpable.

- Grado 1.- No hay apariencia visual de cresta pero se puede palpar un ligero acumulo adiposo.

- Grado 2.- Cresta apreciable visualmente, pero el acumulo de grasa se deposita equitativamente desde la nuca a la cruz. La cresta se puede palpar y se inclina de un lado a otro.

- Grado 3.- Cresta engrosada y más grande. La grasa se deposita mayoritariamente en el medio del cuello dando una apariencia del montículo. La cresta se palpa en una mano y empieza a perder flexibilidad hacia los lados.

- Grado 4.- Cresta muy grande y engrosada que no es posible palpar con una mano por su gran tamaño, se mueve fácilmente de un lado a otro. La cresta puede tener arrugas o pliegues perpendiculares a la línea superior.

- Grado 5.- La cresta es tan grande que se cae permanentemente hacia un lado.
Medidas del cuello: Se recomienda medir la distancia de una línea recta desde la nuca a su borde craneal de la cruz. La circunferencia del cuello se debe medir perpendicularmente a esta línea al 25, 50 y $75 \%$ de la distancia entre estas dos estructuras, las cuales se les ha designado como D1, D2, y D3 respectivamente. La circunferencia media del cuello se puede calcular mediante la media de estas tres medidas ${ }^{11,12}$. Existen medidas estándar de acuerdo a las razas del largo y diámetro del cuello. La evaluación de cuello en el examen precompra del caballo de Pura Raza Española, requiere especial atención. La cresta del cuello afecta a un largo numero de caballos de Pura Raza Española y otros tipos de caballos de razas "Barrocas" con un largo cuello y abundantes crines (Lusitano, Morgan, Paso fino, entre otros $)^{13}$. El Reglamento de Concursos Morfológicos-Funcionales de Caballos y Yeguas de Pura Raza Española Año 2013 (Asociación Nacional de Criadores de Caballo de Pura Raza Española), recientemente actualizado en $2016^{14,15}$. Se describe a continuación: Características generales: I.- Eurimétrico mesolineo y de perfil convexo a recto, de conformación proporcionada, notable armonía y de gran belleza con apreciable dimorfismo sexual. El cuello: debe ser de tamaño y longitud medios, ligeramente arqueado y musculado (manos, en las yeguas). Bien insertado en la cabeza y tronco. Crin abundante y sedosa (Asociación Nacional de Criadores de Caballo de Pura Raza Española, 2016) ${ }^{15}$. En caso de la presencia de la deformación del borde dorsal del cuello estos caballos deben ser inhabilitados y descalificados del libro genealógico de la raza, debe ser notificado al comprador y reportado en el informe de compra-venta.

\section{Métodos invasivos}

Aspirado con Aguja Fina: esta técnica representa una herramienta para la valoración de la región dorsal del cuello, con mínimas com- 
plicaciones ${ }^{16}$. Una vez realizada la respectiva antisepsia requerida, con una aguja $\mathrm{N}^{\circ} 21 \times 1.5$ Gaus, la técnica consiste en realizar una punción en la región dorsal del cuello a $4 \mathrm{~cm}$. del inicio de la cresta, aspirados: craneal, medio y lateral, tanto de lado izquierdo como de lado derecho. Se realiza un extendido en una lámina porta objeto, se fija y una vez teñida se realiza la observación al microscopio óptico. Se realiza una estimación de acuerdo a la valoración de los elementos celulares predominantes ${ }^{16}$ :

- Grado 0: No se observan adipocitos, sólo fibras de colágeno y escasas células fusiformes uniformes.

- Grado 1: Se observaron escasos adipocitos, fibras de colágeno predominantes y células fusiformes uniformes abundantes.

- Grado 2: se caracterizaron por una mezcla de adipocitos maduros, células fusiformes uniformes y fibras de colágeno.

- Grado 3: se observan adipocitos maduros, células fusiformes poco uniformes y fibras de colágeno escasas.

- Grado 4: se observaron adipocitos maduros y células fusiformes uniformes, ausencia de fibras de colágeno.

- Grado 5: se caracterizaron por sólo adipocitos maduros.

No es común observar células inflamatorias, mitosis, atipias, células mastociticas, vasos sanguíneos o matriz mixoide. Estos elementos pueden estar asociados a una condición patológica subyacente.

Biopsia Modificada: La técnica de biopsia modificada de la región dorsal del cuello en équidos consiste en la toma de muestras mediante un sistema de biopsia ajustable, se colectan 6 muestras (punch), en la región dorsal del cuello (craneal, media y caudal), del lado derecho e izquierdo, con una profundidad de $2-3 \mathrm{~cm} .{ }^{17}$. Las muestras se deben identificar en una solución de formol al $10 \%$ y posteriormente procesados por los métodos convencionales histológicos teñidos con la coloración de rutina hematoxilina y eosina. Las biopsias deben ser clasificadas por los patrones de reconocimiento histológicos descritos en la literatura para caballos ${ }^{18}$.

- Grado 0.-Se observan solo fibras musculares, no se observan vacuolas grasas.

- Gradol.-Se observa un escaso depósito adiposo. Se observa un tejido muscular inalterado.

- Grado 2.- Se evidencian vacuolas de grasa en el tejido muscular y en el espacio intermiofibrilar, propenso a la coalescencia.

- Grado 3.- Se observan abundantes vacuolas de grasa en el espacio intermiofibrilar con tendencia a coalescer y escasa infiltración de grasa en el tejido muscular (lipomatosis moderada).

- Grado 4.-Se evidencian abundantes vacuolas de grasa en el espacio intermiofibrilar con tendencia a coalescer e infiltración grasa en el tejido muscular (lipomatosis marcada).

- Grado 5.- Sólo se observan vacuolas de grasa, ausencia de tejido muscular (lipomatosis severa).

Análisis morfométrico: el análisis morfometrico se puede realizar mediante programas de procesamiento de imágenes digitalizadas de las preparaciones histológicas a partir de las biopsias del cuello, como el programa Imagen Tools. Una vez calibrado el programa, se puede maximizar la imagen de interés y medir las variables (superficie y profundidad). En cada medición se registran los datos morfométricos correspondientes a las variables en una hoja de resultados del programa y automáticamente se obtiene la media y la desviación estándar de las mediciones realizadas, acompañado de un estudio estadístico permite un estudio objetivo $^{16}$.

Adicionalmente es posible emplear la tinción de Tricromico de Masson, e inclusive la coloración especial Oil Red, así como otras técnicas de diagnostico como la Inmunohistoquimica con los anticuerpos: Desmin, Vimentin, ALT/WDLS, MDM2M, CDK4 ${ }^{16}$. El estudio ultraestructural a través de la micros- 
copia electrónica puede realizarse con algunos hallazgos interesantes como alteraciones de las fibras musculares y la presencia de gotas lipidicas, por lo común se realiza con fines de investigación pero tienen una gran significancia diagnostica ${ }^{16}$.

\section{VALORACIÓN CRÍTICA}

La compra de un caballo puede ser un negocio arriesgado. El vendedor, en la mayoría de los casos, tiene un conocimiento profundo de los rasgos del caballo particular que es vendido y generalmente un comprador tendrá solamente una oportunidad limitada de la inspección ${ }^{1}$. En las circunstancias inherentes a los defectos en un caballo en particular son a veces difíciles de detectar. La edad representa un reto diagnostico importante en cuanto a la deformación del borde dorsal del cuello, ya que esta condición por lo común se presenta a partir de los 6 años. La edad en la cual se realiza la venta de caballos finalizando el proceso de doma, donde el valor económico se incrementa de manera proporcional a su rendimiento. Es por ello la necesidad de desarrollar métodos diagnostico predictivos, para la deformación del borde dorsal del cuello en las razas con mayor predisposición, aunque muchos factores genéticos y ambientales pueden estar asociados directamente y determinar el desarrollo de la deformación del borde dorsal en el caballo. El costo de realizar pruebas adecuadas a menudo es prohibitivo, a menos que el caballo en cuestión sea especialmente valioso. Se aprecia que ciertas condiciones no son detectables en absoluto o son lo suficientemente raras como para no justificar una investigación especial. Si bien los vendedores y los compradores suelen obtener informes previos a la compraventa, los litigios contra los veterinarios, en la medida en que se han producido tales informes, se han iniciado casi sin excepción en nombre de un comprador afectado ${ }^{19}$. La evaluación del cuello en el examen precompra del caballo, requiere especial atención princi- palmente en las razas "Barrocas" incluyendo al caballo de Pura Raza Española, Lusitano, Morgan, Paso fino, así como Ponies y Minihorses, e inclusive Hispano-Árabe y caballos cruzados. Dada las importantes pérdidas económicas que representa para un propietario o criador la depreciación asociada a la cresta del cuello o deformación del borde dorsal del cuello (cuello de gato), en la que se descalifica y se excluye del registro del libro genealógico, es necesario considerar métodos diagnósticos precisos que garanticen la compra del caballo y el futuro desempeño atlético.Las lesiones del cuello son una causa primaria poco común de dolor, ya sea representado en la claudicaciones o pobre rendimiento atlético, pero se debe considerar si las técnicas analgésicas locales de los miembros no pueden abolir la claudicación o si hay signos clínicos atribuibles directamente al cuello, como el dolor, postura anormal del cuello, rigidez o sudoración irregular. El diagnóstico preciso requiere de un cuidadoso examen clínico, la exclusión de otras causas de claudicación o pobre rendimiento, y la interpretación precisa de los resultados de diagnóstico por imágenes ${ }^{21}$. La anatomía detallada de las articulaciones cervicales y de procesos articulares, ha recibido poca atención en la literatura, sin embargo estos trastornos se han relacionado con la compresión de la médula espinal que resulta en signos clínicos graves, como la ataxia y la debilidad ${ }^{21}$. En un reciente estudio por imagen las anomalías más representativas encontradas en las radiografías fueron la subluxación dorsal o inestabilidad vertebral, la osteoartritis de los procesos articulares y las fracturas vertebrales de distinta naturaleza ${ }^{6}$. Los diagnósticos más frecuentes obtenidos a partir del estudio radiográfico y clínico fueron la mielopatía estenótica cervical (MEC), y la osteoartritis cervical $(\mathrm{OA})^{7,11}$. Es necesario mencionar la condición de obesidad y el síndrome metabólico equino que representa un importante riesgo de disfunción endocrina con la subsecuente resistencia a la insulina $y$ laminitis. Si bien cada veterinario tiene el obje- 
tivo de realizar cada examen previo a la compra de forma precisa y exhaustiva, no es posible dentro de los límites del examen detectar todos los posibles problemas o fallos potenciales. El concepto erróneo más común con respecto al examen previo a la compra de equinos es que una vez realizado, independientemente de los hallazgos reportados por el veterinario, un certificado de examen representa alguna forma de garantía de que el caballo permanecerá libre de problemas una vez comprado ${ }^{19}$. El veterinario no debe hacer ninguna determinación y no expresar ninguna opinión sobre la idoneidad del animal para el propósito previsto. Esta cuestión es un juicio de negocios que es exclusivamente responsabilidad del comprador que él o ella debe hacer sobre la base de una variedad de factores, sólo uno de los cuales es el examen y el informe proporcionado por el veterinario. Es importante hacer énfasis que todos los exámenes realizados requieren la autorización del vendedor y el consentimiento y aprobación de las partes interesadas. En conclusión describimos, mediante una revisión crítica, los métodos recomendados y las herramientas disponibles para la evaluación del cuello en el examen precompra de caballos.

\section{REFERENCIAS}

1. Dulai Wenholz S. 2007. Understanding the Prepurchase Exam. Disponible en http:// www.thehorse.com/articles/19105/understanding-the-prepurchase-exam

2. Examen precompra. Clínica La Sierra. Disponible en: http://clinicaequinalasierra.com/ servicios-veterinarios/examen-precompra/

3. Norton J. 2016. The Buyer's Guide to Prepurchase Exams. The horse. Disponible en http://www.thehorse.com/articles/34952/ the-buyers-guide-to-prepurchase-exams

4. Examen Precompra: evaluación y valoración del riesgo de los hallazgos radiográficos. 2016. V Congreso Anual de la A.V.E.E., 2-3 Junio.
5. Figley S. 2017. Disponible en: https://www. horsejournals.com/popular/horse-industry/ pre-purchase-exam

6. Pre-Purchase Examinations. Disponible en: https://www.rossdales.com/services/sportand-leisure-horses/pre-purchase-examinations

7. Morales A, Méndez A, Pérez-Arévalo J. 2014. La region del cuello del caballo. Connotaciones anatomo-aplicativas. Una revision. Int. J. Morphol. 32: 4. 1212-1221.

8. Díez, E.; Sánchez de Medina, A. \& Novales Durán, M. 2010. Aportaciones de la radiología digital al estudio de las patologías cervicales del caballo. Equinus, 27:53-68.

9. Rose R.J., Hodgson D. 1995. Manual of equine practice. $\mathrm{Wb}$ Sanders.Company, Harcourt Brace Jovanovich. Inc. Philadelphia, Pennsylvania, U.S.A. 130-132.

10. Carter RA, Geor RJ, Staniar WB, et al. 2009. Apparent adiposity assesed by Standardised scoring systems and morphometric measurements in horses and ponies. Vet $\mathrm{J}$ : 179 (2):204-210.

11. Diez de Castro E, Pineda Martos C, Martin Cuervo M, Quintero Felices S, Mendez Vazquez N, Aguilera Tejero E. 2012. Valoracion de obesidad y resistencia a la insulina en el Caballo de Pura Raza Española (PRE). Equinus: 34 Tercer Cuatrimestre: 58-75.

12. Frank N, Elliot SB, Brant LE and Keisler DH. 2006. Physical characteristics, blood hormone concentration, and plasma lipid concentrations in obese horses with insulin resistance. J Am Vet Med Assoc: 228 (9): 1983-1390.

13. Sánchez M, Azor P, Molina A,Parkin T,Rivero J, Valera M. 2016.Prevalence, risk factors and genetic parameters of cresty neck in PuraRazaEspañol horses. EquineVet J. Feb.

14. Asociación Nacional de Criadores de Caballo de Pura Raza Española 2013. Reglamento de Concursos Morfológicos-Funcionales de Caballos y Yeguas de Pura Raza Españo- 
la. Disponible en: http://www.ancce.es/nueva/ver-noticia/884/aprobado-el-reglamentode-concursos-morfologicos-funcionales-decaballos-y-yeguas-de-pura-raza-espanolapara-2013/26/ PDF 46-57. 2013.

15. Asociación Nacional de Criadores de Caballo de Pura Raza Española. Reglamento de Concursos Morfológicos-Funcionales de Caballos y Yeguas de Pura Raza Español. Disponible en: http://www.ancce.es/_docs/ documentos/2016/Morfologico/reglamento2016_web.pdf. PDF69-73 2016.

16. Morales Briceño A, Méndez Sánchez A, Méndez-Angulo J, Escamilla-Sánchez A, Pérez-Arévalo J. 2017. Diagnostic tools for the study of the cresty neck in horses.Intern J Appl Res Vet Med. 15:1. 52-60.

17. Morales-Briceño A, Escamilla-Sánchez A, Méndez-Sánchez A, Pérez-Arévalo A, Méndez-Angulo J.2016. Poster D 28: Modified biopsy technique and histopathological grades for cresty neck in Spanish Purebred horse. Annual Meeting American College of Veterinary Pathologist.New Orleans, Louisiana, USA.

18. Morales A, Escamilla Sánchez A, Méndez Sánchez A, Méndez J, Pérez Arévalo J. 2017. Histopathological pattern recognition of cresty neck in horses in Spain.Volumen: Braz J Vet Pathol. 10(1).170-174.

19. A guide to examination of horses. 2006. Equine Veterinarians Australia (EVA) Members Handbook. Fourt Edition.172.

20. Dyson, S. J. 2011. Lesions of the equine neck resulting in lameness or poor performance. Vet. Clin. North Am. Equine Pract., 27(3):417-37.

21. Claridge, H. A.; Piercy, R. J.; Parry, A. \& Weller, R. 2010. The 3D anatomy of the cervical articular process joints in the horse and their topographical relationship to the spinal cord. Equine Vet. J., 42(8):726-31. 